\title{
An MRI-compatible defibrillator: initial testing in volunteers and swine
}

\author{
Ehud J Schmidt ${ }^{1 *}$, Ronald D Watkins², Menekhem M Zviman, Michael Guttman ${ }^{3}$, Wei Wang ${ }^{1}$, Henry Halperin ${ }^{3}$ \\ From 19th Annual SCMR Scientific Sessions \\ Los Angeles, CA, USA. 27-30 January 2016
}

\begin{abstract}
Background
Performing MRI-guided interventions, such as for the treatment of ventricular tachycardia, requires the ability to rapidly detect and treat acute cardiac conditions, should they occur in the MRI. We developed MRI-compatible 12lead ECG systems, and demonstrated detection of acute ischemia during imaging [Tse, MRM. '13,Zhang, MRM. ' 15 ], providing a tool to non-invasively detect cardiac events in the MRI. It is presently necessary to remove patients from the MRI to treat cardiac events. This increases risk, consequently restricting MRI imaging, as well as MRI-guided intervention, to lower-risk patients and lower-risk procedures. Defibrillation in the bore could, therefore, accelerate the growth of diagnostic (such as exercise stress-perfusion and acute post-infarct studies) and therapeutic MRI studies. The study objective is to develop a defibrillation system that can safely be used on patients inside a 1.5 Tesla MRI. In addition, this system should not compromise MRI image quality.
\end{abstract}

\section{Methods}

The output of a commercial Zoll Medical (Chelmsford, MA) M-series defibrillation generator (Figure 1A) was connected to a novel 7-pole low-pass filter, designed to reduce by $80 \mathrm{~dB}$ emission of both differential-mode and common-mode $63.8 \mathrm{MHz}$ signals into the scanner, while maintaining the essential $67-\mathrm{KHz}$ defibrillator-pad ON/ OFF detection utility of the generator, and its ECG monitoring utility. The low-pass filter was connected to commercial Zoll multifunction defibrillator pads on a subject's chest via 3-meter-long high-voltage twisted-pair cables, mounted with tuned RF-traps ("Baluns") at 30-cm increments (Figure 1B). Volunteer cardiac SSFP imaging was performed on a Siemens 1.5T Aera using the scanner's cardiac array, with the generator in the MRI suite, but outside the 5-Gauss line. Image quality was compared under three conditions; with (a) the generator OFF, (b) generator ON in ECG-monitoring mode, powered from the on-board battery, or (c) the A/C power outlet. In addition, multiple full-power (2000V) defibrillations were performed on a swine, inside the MRI, using the system.

\section{Results}

A slight 2 and $1 \mathrm{~dB}$ reduction in image SNR was observed (Figure $1 \mathrm{C}$ ) with the system running on $\mathrm{A} / \mathrm{C}$ power, and running on battery, respectively, relative to the system OFF. The system OFF state displayed no discernable SNR penalty relative to removal of the system from the room. Image artifacts from the defibrillator pads extended only $\sim 6 \mathrm{~mm}$ below the skin. Defibrillator-pad heating did not occur. The swine defibrillations were completed without any complications.

\section{Conclusions}

Defibrillation inside the MRI may be performed with a modified commercial defibrillator system, enabling the performance of higher-risk imaging and MRI-guided interventions.

\section{Authors' details}

${ }^{1}$ Brigham and Womens Hospital, Boston, MA, USA. ${ }^{2}$ Radiology, Stanford, Stanford, CA, USA. ${ }^{3}$ Cardiology, Johns Hopkins, Baltimore, MD, USA.

Published: 27 January 2016

doi:10.1186/1532-429X-18-S1-0122

Cite this article as: Schmidt et al: An MRI-compatible defibrillator: initial testing in volunteers and swine. Journal of Cardiovascular Magnetic Resonance 2016 18(Suppl 1):0122. 


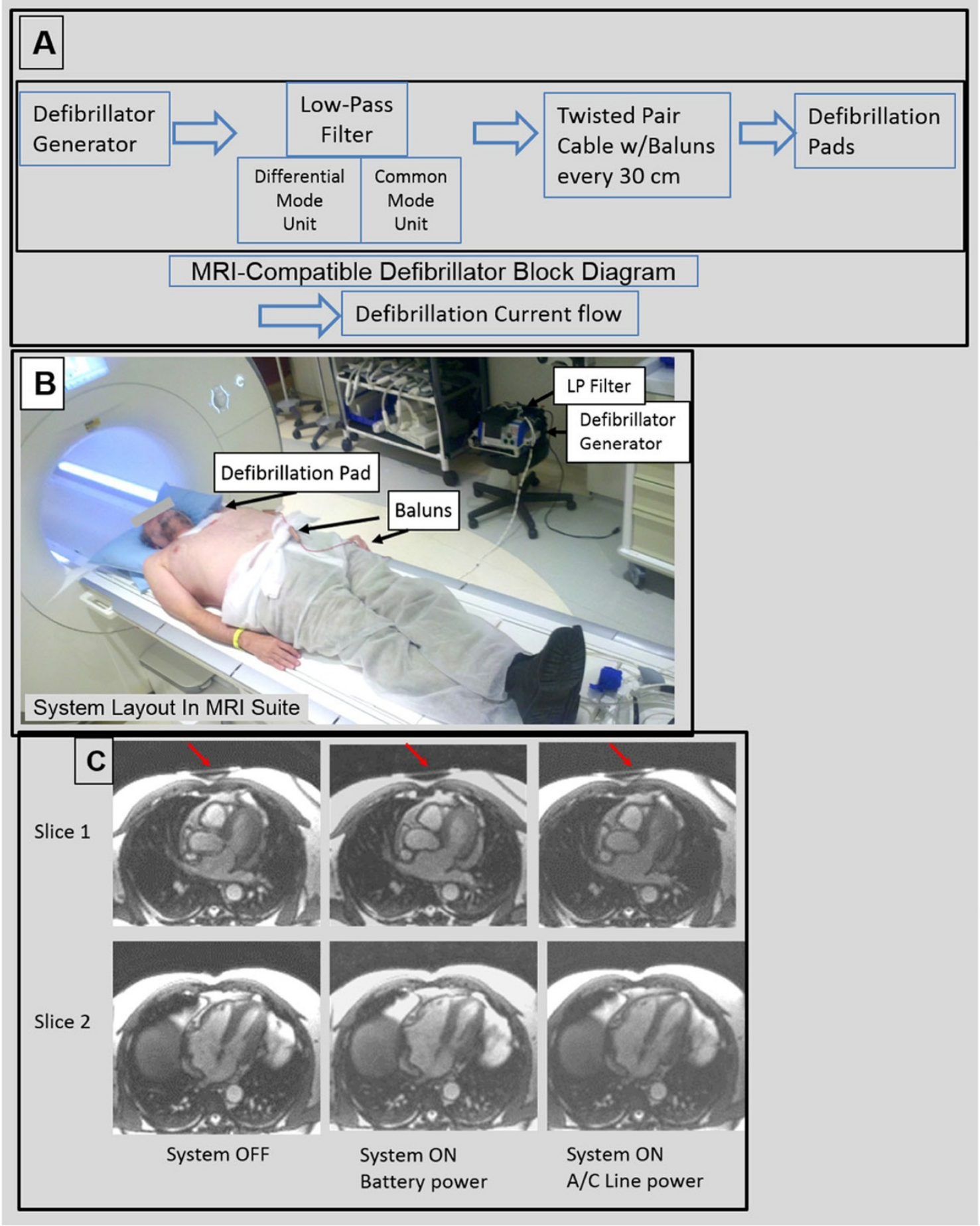

Figure 1 (A) Block diagram of MRI-compatible defibrillator. 7-pole Low-pass filter has diffential-mode and common-mode units to maximally suppress unwanted RF current induction into MRI. (B) The layout of the system inside the MRI room. (C) 1.5T MRI SSFP images with defibrillator generator OFF, generator ON powered from the battery, and generator ON powered from the line (worst SNR condition). Red arrows denote location of defibrillation pads. 